\title{
Anesthetic management of a pregnant patient with significant post-burn scars and contractures
}

\author{
Suman Rajagopalan*, Jaime Ortiz and Shobana Chandrasekhar \\ Department of Anesthesiology, Baylor College of Medicine, Houston, TX, USA
}

\begin{abstract}
Post burn contracture and scarring in obstetric patient is not a very common occurrence. We present this 38-year-old pregnant patient who had extensive post burn scarring involving more than $90 \%$ of the body surface area with restricted mouth opening and severe neck contractures. We discuss the various problems encountered in the management of her urgent Cesarean delivery for severe preeclampsia and non-reassuring fetal status. This includes the issues with airway management, monitoring devices and intravenous access.
\end{abstract}

\section{Introduction}

The advances in acute management of burns have resulted in a significant decrease in mortality; however, the quality of life in survivors continues to be enormously impacted by the high incidence of post-burn scars [1]. The post-burn scars can undergo hypertrophy and result in contractures that affect underlying tissues including, fascia, muscles, tendons, nerves, and blood vessels. The anesthetic management in patients with extensive post-burn scarring is complicated by difficulties with intubation and vascular access, in addition to inadequate ventilation due to decreased compliance of the chest wall. We present the challenges faced while managing a pregnant patient with extensive post-burn scars. Written consent was obtained from the patient to publish this case report.

\section{Case description}

A 38-year-old primigravida was admitted to the hospital at 27 weeks of gestation for the management of severe preeclampsia, elevated aminotransferase levels, and hyperglycemia. At the age six, she had sustained severe burns involving greater than $90 \%$ of the body surface area that mandated a tracheostomy and below knee amputation. The burns resulted in extensive hypertrophic and atrophic scars involving face, neck, chest, abdomen, and contractures of elbows and knees (Figure 1). The movements of the neck were significantly restricted and mouth opening was limited to $2.5 \mathrm{~cm}$. Ultrasound examination revealed fetal intrauterine growth restriction and absent end-diastolic flow of the placental vasculature consistent with placental insufficiency. The blood glucose levels were consistently greater than $400 \mathrm{mg} / \mathrm{dl}$ for which an insulin infusion was started. Two doses of betamethasone were
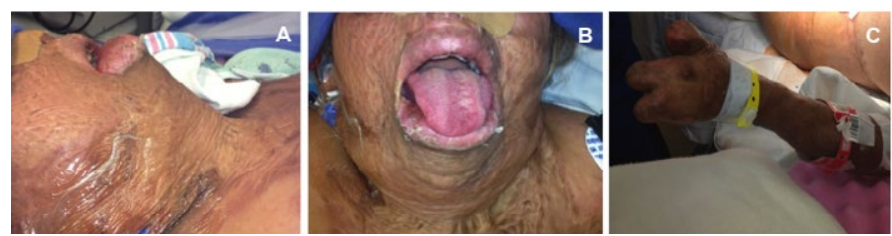

Figure 1. Extensive post burn contractures leading to significantly restricted neck extension (A), limited mouth opening (B), and deformities of the upper extremity (C). administered to foster fetal lung maturity in preparation for delivery. Due to religious beliefs, the patient declined to receive transfusion containing red blood cells but agreed to have albumin, "cell-saver", plasma, or cryoprecipitate transfusions, if needed.

Within a few hours of admission, her fetal monitoring revealed repeated fetal decelerations and non-reactive tracings. The obstetric team decided to proceed with her Cesarean delivery at around midnight. As she was an anticipated difficult airway and the surgical closure was complex due to extensive scaring, the plan was to proceed with general anesthesia. A single 18 gauge peripheral intravenous access was all that could be established due to the thick scars and so the decision was made establish a central venous access through the right internal jugular vein. She had such extensive scaring and contractures that we were worried about the reliability of non-invasive blood pressure monitoring. An arterial line was placed in the left brachial artery under ultrasound guidance as the radial and dorsalis pedis arterial pulses were not palpable due to entrapment of these vessels in the scar tissue. An epidural catheter was placed for post-operative pain management since the lower lumbar region was free of burn scars.

Following pretreatment with non-particulate antacid, topical anesthesia of the oral cavity and pharynx with achieved with lidocaine. The vocal cords were visualized with a fiber-optic laryngoscope and tracheal intubation was successfully performed with a 6.0 endotracheal tube. Given her history of severe preeclampsia and past history of prolonged tracheostomy, a smaller size endotracheal tube was chosen to avoid multiple attempts at intubation. Once the position of the endotracheal tube was confirmed, propofol was used for induction. Anesthesia was maintained with a combination of sevoflurane, nitrous oxide, and cisatracurium. Extreme caution was exercised in

Correspondence to: Suman Rajagopalan, Department of Anesthesiology, Baylor College of Medicine, One Baylor Plaza, MS: BCM-120, Houston, Texas 77030, USA, Tel: +1-713-873-2860; Fax: 713-873-2867; E-mail: srajagop@bcm.edu

Key words: post burn contracture, pregnancy, cesarean section, difficult intubation Received: March 08, 2015; Accepted: March 25, 2015; Published: March 27, 2015 
positioning the patient due to multiple contractures. A live male baby was delivered with APGARs of 1 and 4 at 1 and 5 minutes, respectively and was immediately transferred to the neonatal intensive care unit for management of prematurity-related complications. Following closure of the uterus, the trauma surgery team assisted with the complex surgical closure of the abdominal wall. The total estimated blood loss was $900 \mathrm{ml}$ and no blood products were transfused. Extubation was uneventful and she recovered well enough to be discharged four days after the surgery.

\section{Discussion}

Few reports exist regarding the management of obstetric patients with old burns and deformities, and its anesthetic implications. The prevalence of pathological scarring post burns is reported to be $77 \%$ with about $28 \%$ developing hypertrophic scar with contractures [1]. The risk of scarring is found to be higher in patients with a higher percentage of burnt surface area and in those with full thickness burns. Depending on the location, there could be various issues during the anesthetic management of these patients.

Scarring around the mouth and neck can lead to limited mouth opening, restricted neck movement, underdeveloped jaw, and distortion of the larynx which present challenges with mask ventilation and intubation [2,3]. Patients with history of inhalational injury and tracheostomy can develop tracheal stenosis that may prevent advancement of endotracheal tube at the site of stenosis and hence require smaller endotracheal tubes [4]. Invasive airway techniques for securing the airway such as cricothyrotomy or tracheostomy are typically difficult due to alterations in the anatomy of the neck and inability to palpate the landmarks.

Pregnancy, in itself, leads to numerous anatomic and physiological changes including capillary engorgement and mucosal edema of the oropharynx, larynx and trachea leading to a more difficult airway. The gravid uterus shifts the position of the stomach and angle of the gastroesophageal junction resulting in an incompetent sphincter. Along with the progesterone mediated smooth muscle relaxant effect on the lower esophageal sphincter, the risk of reflux and subsequent pulmonary aspiration increases [5]. The incidence of failed obstetric intubation is widely reported to be $\sim 1$ in 270 patients, which is higher than in a non-pregnant patients [6,7]. Careful planning is thus necessary when an obstetric patient presents with anticipated difficult intubation, as both the mother and baby are at-risk for anesthetic and operative complications. The risk of failed intubation and its associated complications for cesarean deliveries could be avoided by using neuraxial techniques; however sometimes the spinal/epidural may be patchy or may result in inadvertent complications. Converting to general anesthesia in an anticipated difficult airway situation, after the start of the surgical procedure, would be suboptimal. In our patient, given the history of extensive abdominal scarring and an anticipated delay with the complex abdominal closure, spinal anesthesia was not preferred. We decided it was safer to proceed with general anesthesia in a controlled elective manner and secure her airway with an awake fiberoptic intubation.

The intravenous access in post burns patients could be challenging due to extensive scarring. Obtaining a non-invasive blood pressure reading can also be difficult depending on the location of burns. Our patient had extensive scarring and contractures of both the upper and lower extremities making it difficult to access the veins or palpate the arteries. Given her history of extensive burns, severe preecclampsia, concern for considerable blood loss, we decided to proceed with the central venous and arterial cannulations under ultrasound guidance $[8,9]$.

Anesthetic drugs except for succinylcholine can be safely used in patients with chronic burns. An increase in thiopental requirement was noted in children for more than 1 year after major burn but no information is available on the effect of propofol following chronic burns. The proliferation of extrajunctional receptors may return to normal levels once the wound heals and protein catabolism subsides. The healing process may take 1-2 years or longer after wound coverage in patients with major burns ( $80 \%$ body surface area) [10]. If the immobilization or muscle catabolism persists secondary to severe contractures, then the up-regulated extrajunctional receptors may not normalize and can predispose to hyperkalemia and cardiac dysrhythmias with succinylcholine.

In conclusion, obstetric patients with childhood burns can present with multiple challenges related to excessive scarring and contractures depending on the extent of burn injuries. Anticipated airway issues along with difficult spinal or epidural placement, inability to obtain intravenous and arterial access, complex surgical closure are a few problems associated with chronic burn contractures. Early referral, careful planning, detailed discussion addressing the issues, both with the patient and the surgical team, lead to a good outcome in our case.

\section{References}

1. Gangemi EN, Gregori D, Berchialla P, Zingarelli E, Cairo M, et al. (2008) Epidemiology and risk factors for pathologic scarring after burn wounds. Arch Facial Plast Surg 10: 93-102. [Crossref]

2. Nahlieli O, Kelly JP, Baruchin AM, Ben-Meir P, Shapira Y (1995) Oro-maxillofacial skeletal deformities resulting from burn scar contractures of the face and neck. Burns 21: 65-69. [Crossref]

3. Han TH, Teissler H, Han RJ, Gaines JD, Nguyen TQ (2012) Managing difficult airway in patients with post-burn mentosternal and circumoral scar contractures. Int J Burns Trauma 2: 80-85. [Crossref]

4. Sarper A, Ayten A, Eser I, Ozbudak O, Demircan A (2005) Tracheal stenosis after tracheostomy or intubation: review with special regard to cause and management. Tex Heart Inst J 32: 154-158. [Crossref]

5. Munnur U, de Boisblanc B, Suresh MS (2005) Airway problems in pregnancy. Crit Care Med 33: S259-268. [Crossref]

6. Hawthorne L, Wilson R, Lyons G, Dresner M (1996) Failed intubation revisited: 17-yr experience in a teaching maternity unit. Br J Anaesth 76: 680-684. [Crossref]

7. Barnardo PD, Jenkins JG (2000) Failed tracheal intubation in obstetrics: a 6-year review in a UK region. Anaesthesia 55: 690-694. [Crossref]

8. Shiloh AL, Eisen LA (2010) Ultrasound-guided arterial catheterization: a narrative review. Intensive Care Med 36: 214-221. [Crossref]

9. Karakitsos D, Labropoulos N, De Groot E, Patrianakos AP, Kouraklis G, et al. (2006) Real-time ultrasound-guided catheterisation of the internal jugular vein: a prospective comparison with the landmark technique in critical care patients. Crit Care 10: R162. [Crossref]

10. Jeevendra Martyn JA, Fukushima Y, Chon JY, Yang HS (2006) Muscle relaxants in burns, trauma, and critical illness. Int Anesthesiol Clin 44: 123-143. [Crossref]

Copyright: (C2015 Cascella M. This is an open-access article distributed under the terms of the Creative Commons Attribution License, which permits unrestricted use, distribution, and reproduction in any medium, provided the original author and source are credited. 